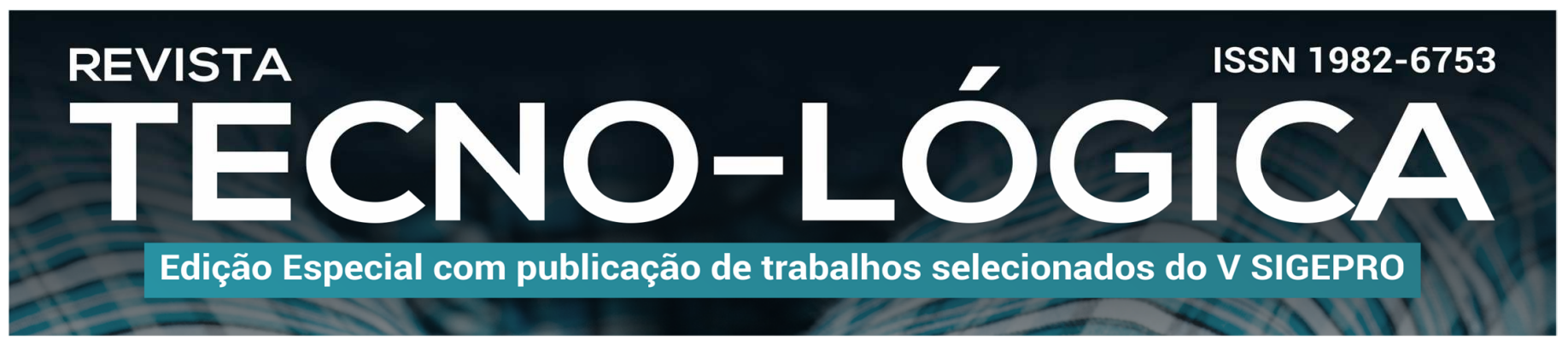

\title{
IMPACTO DAS TECNOLOGIAS DIGITAIS DA INDÚSTRIA 4.0 NA ÁREA DE SAÚDE E SEGURANÇA DO TRABALHO: UMA ANÁLISE ATRAVÉS DA REVISÃO SISTEMÁTICA DA LITERATURA
}

\author{
Jocieli Francisco da Silva ${ }^{1 *}$, Flávia Luana da Silva ${ }^{1}$
}

1 Programa de Pós-Graduação em Engenharia de Produção e Sistemas, Universidade do Vale do Rio dos Sinos (UNISINOS), 93022-750, São Leopoldo, Brasil.

*E-mail: jocielifs@outlook.com

Recebido em: $17 / 09 / 2020$ Aceito em: 06/11/2020 DOI: 10.17058/tecnolog.v2i0.15565

\section{RESUMO}

O novo paradigma da indústria 4.0 está revolucionando as organizações e tem impacto em diferentes setores. Olhando por este espectro, o presente trabalho tem como objetivo verificar qual o impacto das tecnologias da indústria 4.0 na área de saúde e segurança do trabalho. Para isso foi realizada uma revisão sistemática da literatura, buscando analisar quais as vantagens, desvantagens e desafios do uso das tecnologias da indústria 4.0 no contexto da saúde e segurança do trabalho. As bases de dados utilizadas foram Web os Science e Scopus e os documentos foram selecionados conforme critérios pré-definidos. Os resultados encontrados apontam que o cenário ainda é muito vago, há poucas pesquisas que abordam o tema tecnologias da indústria 4.0 na área de segurança do trabalho evidenciando uma lacuna de pesquisa e exploração científica.

Palavras-chave: Saúde e Segurança do Trabalho. Indústria 4.0. Revisão da Literatura.

\section{Introdução}

A saúde é definida pela Organização Mundial da Saúde (OMS) como um estado de completo bem-estar físico, mental e social, além da ausência de doenças. Ela está presente em um contexto amplo, incluindo o ambiente social, econômico, físico e as características e comportamentos individuais das pessoas [1].

Em relação a garantia da saúde no ambiente de trabalho é de incumbência da área de saúde e segurança do trabalho garantir a saúde e integridade física dos trabalhadores no ambiente em que atuam [2].

Os acidentes de trabalho devem ser eliminados, garantindo que todos os locais de trabalho sejam seguros. Acidentes de trabalho tem um custo humano, social e econômico significativo [3]. Segundo estimativas da organização nacional do trabalho (OIT), cerca de 2,3 milhões de pessoas morrem e 300 milhões ficam feridos todos os anos no mundo em acidentes de trabalho $[4]$. 


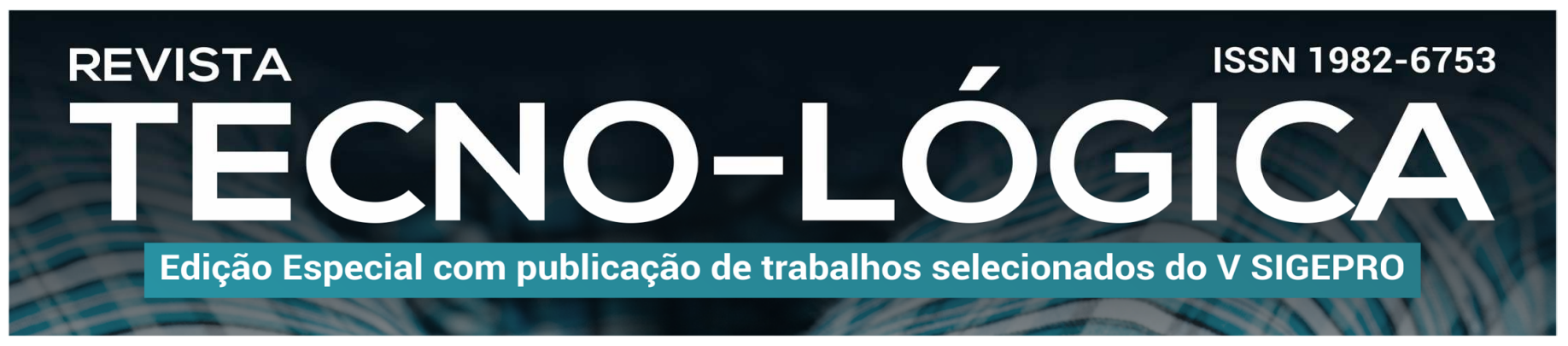

Um dos paradigmas atuais estudados é a quarta revolução industrial, comumente chamada de indústria 4.0, que surgiu no ano de 2011 na Alemanha e aborda a introdução de tecnologias nos processos produtivos [5]. Este paradigma tem como um dos objetivos principais contribuir para a melhoria da segurança no local de trabalho [6].

A transição para a Indústria 4.0 mudará drasticamente a forma como humanos e máquinas colaboram, resultando em novas demandas sobre como o trabalho é realizado de forma segura e eficaz. Alguns autores buscam examinar as ameaças que estão surgindo na era da Indústria 4.0. Acredita-se que vários riscos sejam consequência da interconexão de sistemas e máquinas, o uso crescente de sistemas digitais, a quantidade e qualidade dos dados recebidos que necessitam ser integradas e analisadas [7].

Acredita-se que a introdução das tecnologias da indústria 4.0 traz consigo, além das tecnologias emergentes, problemas que podem afetar a área de saúde e segurança no trabalho, tais como aumento no índice de stress dos funcionários, as ameaças de perda de emprego criadas pela automação da era digital, dificuldades na interação homem-máquina, entre outros [8-9]. Já Contreras et al.[10] afirmam o oposto, argumentando que tecnologias como à inteligência artificial foram desenvolvidas para tornarem as entidades inteligentes e garantirem o enriquecimento da linha de produção. A inteligência artificial vem sendo empregada na criação de robôs, utilizados nos processos de coleta e armazenagem, além de veículos autônomos e drones, utilizados para entregas de materiais em locais de risco. Na maioria dos casos, estas soluções estão relacionadas ao aumento da segurança das operações [11].

Há autores que apontam as vantagens na introdução das tecnologias da indústria 4.0 na área de segurança do trabalho e há os autores que apontam as desvantagens. Diante desse contexto, o presente trabalho tem como objetivo contribuir para preencher essa lacuna, buscando identificar qual o impacto das tecnologias da indústria 4.0 para a área de segurança do trabalho. Para alcançar este objetivo o método utilizado foi a revisão sistemática da literatura.

\section{Materiais e Métodos}

Tendo em vista o objetivo deste artigo o método proposto para sustentar o estudo foi a revisão sistemática da literatura. Os bancos de dados selecionados para a pesquisa foram Web of Science e Scopus. A pesquisa foi realizada no dia 06 de janeiro de 2020, foram considerados todos os tipos de documentos, com a linguagem em inglês e português e sem período específico. As palavras-chaves utilizadas para consulta foram definidas depois de uma pesquisa exploratória em artigos sobre revisão sistemática da literatura para os temas indústria 4.0 e saúde e segurança do trabalho e são apresentadas no quadro 1 . O filtro de busca foi que as palavras estivessem presentes no título, resumo ou palavraschaves do artigo. O operador booleano "and" foi usado para permitir a combinação dos grupos de palavras a serem pesquisadas e o operador "or" foi utilizado entre os grupos, uma vez que, as palavras são sinônimas e o objetivo é trazer todos os documentos que tratem do assunto.

A matéria publicada nesse periódico é licenciada sob forma de uma Licença Creative Commons - Atribuição 4.0 Internacional http://creativecommons.org/licenses/by/4.0/

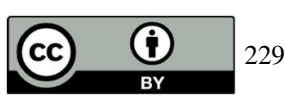




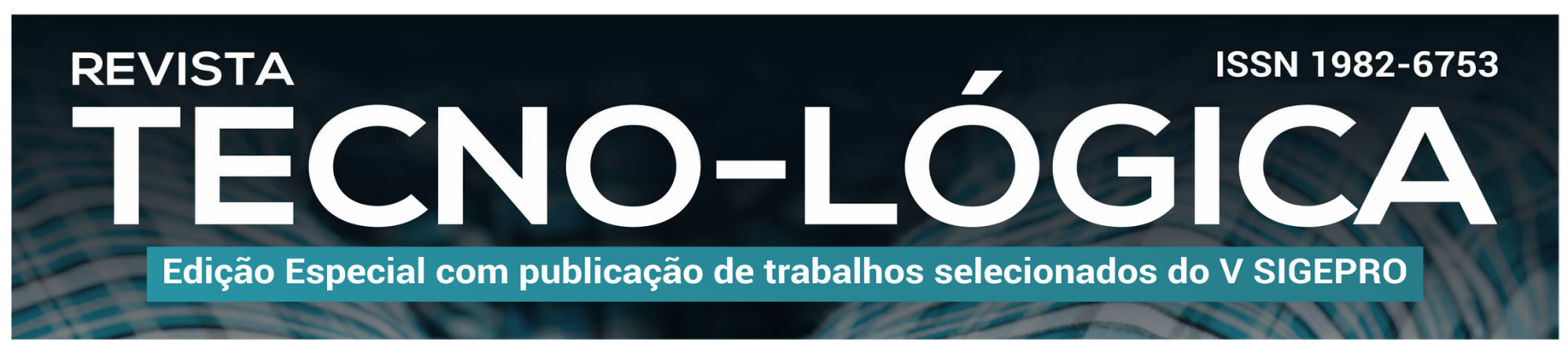

Quadro 1: resultados da pesquisa nas bases Scopus e Web of Science. Fonte: elaborado pelos autores (2020).

\begin{tabular}{|c|c|c|}
\hline Database & Termos de pesquisa & $\begin{array}{l}\text { Documentos } \\
\text { encontrados }\end{array}$ \\
\hline Scopus & $\begin{array}{l}\text { ("industry 4.0" OR "4th Industrial Revolution" } \\
\text { OR "digital manufacturing" OR "digital } \\
\text { enterprise" OR "smart manufacturing") AND } \\
\text { ("Occupational safety and health" OR "safety } \\
\text { culture" OR "safety climate" OR "safety } \\
\text { management systems" OR "Total Worker } \\
\text { Health" OR "Organizational culture") }\end{array}$ & 31 \\
\hline $\begin{array}{l}\text { Web of } \\
\text { Science }\end{array}$ & $\begin{array}{l}\text { ("industry 4.0" OR "4th Industrial Revolution" } \\
\text { OR "digital manufacturing" OR "digital } \\
\text { enterprise" OR "smart manufacturing") AND } \\
\text { ("Occupational safety and health" OR "safety } \\
\text { culture" OR "safety climate" OR "safety } \\
\text { management systems" OR "Total Worker } \\
\text { Health" OR "Organizational culture") }\end{array}$ & 27 \\
\hline
\end{tabular}

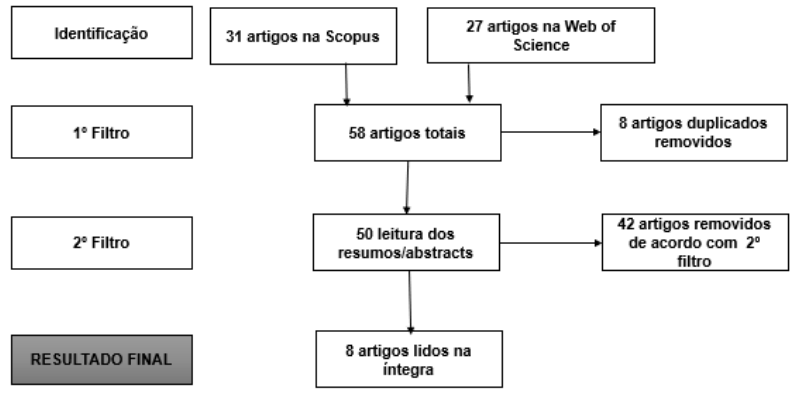

Figura 1 - Processo de seleção dos artigos. Fonte: elaborado pelos autores (2020).

$\mathrm{Na}$ seção seguinte serão especificados os métodos definidos para o agrupamento e a classificação dos artigos escolhidos que compõe a revisão.

\section{Resultados}

O pouco número de documentos resultantes da revisão

Após esse filtro os dados foram consolidados para serem aponta que há pouca discussão em relação ao impacto das tecnologias da indústria 4.0 na área de saúde e segurança do trabalho. O Quadro 2 apresenta a relação dos trabalhos publicados por autores encontrados nesta revisão.

literatura e conforme critérios predefinidos para a seleção do artigos. Os critérios de exclusão dos artigos foram definidos como: (I) artigos repetidos foram excluídos, (II) leitura dos abstracts/resumos dos trabalhos na busca por artigos que abordam indústria 4.0 + segurança no trabalho/no ambiente/ na operação. Após esse filtro, restaram oito documentos para leitura. Esse montante foi inteiramente lido, buscando identificar quais as vantagens e desvantagens da introdução das tecnologias da indústria 4.0 para a área de saúde e segurança do trabalho. A Figura 1 mostra a sequência de etapas descritas acima e o agrupamento dos artigos encontrados em cada uma destas. 


\section{REVISTA}

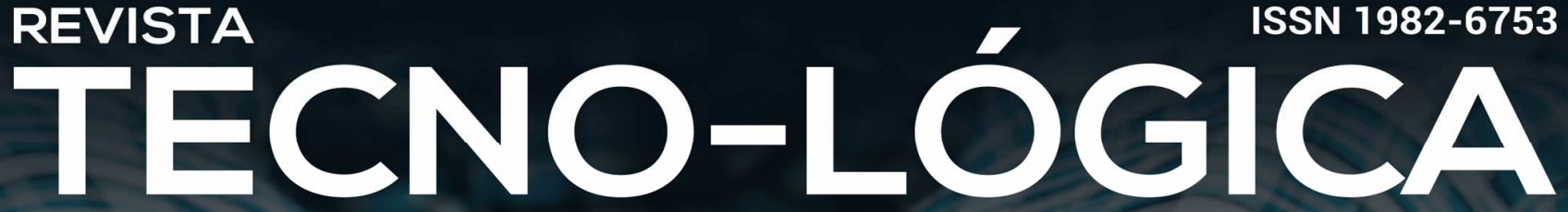

Edição Especial com publicação de trabalhos selecionados do V SIGEPRO

Quadro 2 - Relação de trabalhos por autor. Fonte: elaborado pelos autores (2020).

\begin{tabular}{|c|c|}
\hline Autor & Título \\
\hline $\begin{array}{l}\text { Lazzerini e } \\
\text { Pistolesi [6] }\end{array}$ & $\begin{array}{l}\text { Artificial Bee Colony Optimization to } \\
\text { Reallocate Personnel to Tasks Improving } \\
\text { Workplace Safety }\end{array}$ \\
\hline $\begin{array}{l}\text { Digmayer e } \\
\text { Jakobs [12] }\end{array}$ & $\begin{array}{l}\text { Developing Safety Cultures for Industry 4.0. } \\
\text { New Challenges for Professional } \\
\text { Communication }\end{array}$ \\
\hline Nickel et al. [9] & $\begin{array}{l}\text { Human-system interaction } \\
\text { requirements to improve machinery and } \\
\text { systems safety }\end{array}$ \\
\hline Wichtl et al. [7] & $\begin{array}{l}\text { Improvements of Machinery and Systems } \\
\text { Safety by Human Factors Ergonomics and } \\
\text { Safety in Human-System Interaction }\end{array}$ \\
\hline Sun et al. [14] & $\begin{array}{l}\text { Indoor Air-Quality Data-Monitoring System: } \\
\text { Long-Term Monitoring Benefits }\end{array}$ \\
\hline $\begin{array}{l}\text { Coldwell et al. } \\
\text { [8] }\end{array}$ & $\begin{array}{l}\text { Negative influences of the } 4 \text { th industrial } \\
\text { revolution on the workplace: towards a } \\
\text { theoretical model of entropic citizen behavior } \\
\text { in toxic organizations }\end{array}$ \\
\hline $\begin{array}{l}\text { Issamar e } \\
\text { Roberto [3] }\end{array}$ & $\begin{array}{l}\text { New and emerging occupational risks (NER) } \\
\text { in industry 4.0: Literature review }\end{array}$ \\
\hline Cooper [13] & $\begin{array}{l}\text { The efficacy of industrial safety science } \\
\text { constructs for addressing serious injuries \& } \\
\text { fatalities (SIFs) }\end{array}$ \\
\hline
\end{tabular}

Verifica-se que há uma tendência de crescimento no número de publicações, mas que este ainda é muito incipiente. As primeiras publicações datam de 2017 e a maior concentração está em 2019.

\section{Artigo 1: Artificial Bee Colony Optimization to} Reallocate Personnel to Tasks Improving Workplace Safety

Este artigo apresenta uma abordagem multiobjetivo para realocar o pessoal nas tarefas de trabalho. O objetivo é melhorar a segurança no local de trabalho, mantendo-se baixo tanto os custos quanto o tempo para aprender as novas tarefas atribuídas. Para atender ao objetivo de realocar o pessoal de forma mais adequado a metodologia utilizada foi o algoritmo TOPSIS.

Para condução dos testes foram realizados experimentos envolvendo duas empresas de calçados. A abordagem de otimização foi implementada no MATLAB é um site foi desenvolvido para coletar informações sobre os colaboradores.

Como muitas tarefas da indústria de calçados são feitas à mão e exigem o uso de máquinas perigosas, os trabalhadores estão continuamente expostos a sérios riscos. A gestão de riscos é tipicamente realizada atribuindo-se as tarefas mais perigosas aos funcionários mais experientes, em torno de 15 anos.

$\mathrm{Na}$ abordagem proposta pelo algoritmo a realocação de pessoal é realizada priorizando os riscos. As ações preventivas foram classificadas em três níveis de prevenção. Os dados de cada funcionário sobre fatores humanos e comportamento foram coletados e incluídas no algoritmo.

Como resultados, nos dois cenários no qual o algoritmo foi testado houve uma melhoria de mais de $100 \%$ no índice de segurança dos funcionários com um baixo e temporário aumento de custos. Os riscos se tornam, portanto, menos prejudiciais e menos propensos à medida que as tarefas são atribuídas aos funcionários mais apropriados, considerando seu nível de cautela e não o tempo de serviço. Além disso, o tempo exigido pelos colaboradores para aprender as novas tarefas atribuídas também sofre redução significativa. 


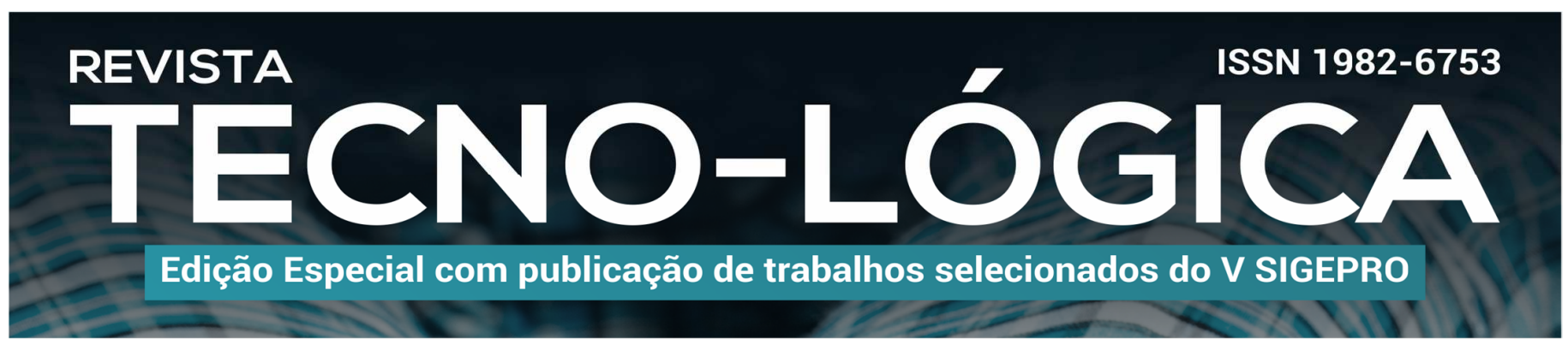

Artigo 2: Developing Safety Cultures for Industry 4.0. New Challenges for Professional Communication

Este artigo apresenta um estudo que visa desenvolver uma abordagem para implantação da Indústria 4.0 em pequenas e médias empresas. O objetivo é desenvolver meios de comunicação e participação que permitam às empresas iniciarem uma transformação da cultura corporativa em relação a aspectos como estruturas de valor, segurança percebida ou comportamento informal.

O objetivo é responder a 3 questões importantes nesse contexto: Quais requisitos devem ser considerados no desenvolvimento de abordagens de transformação para uma cultura de segurança 4.0? Quais os requisitos em relação aos valores precisam ser considerados no desenvolvimento de abordagens de transformação para uma cultura de segurança 4.0? Quais requisitos em relação aos fatos precisam ser considerados no desenvolvimento de abordagens de transformação para uma cultura de segurança 4.0?

Para identificar estes requisitos foram realizados dois estudos de caso, em pequenas empresas metalúrgicas que atualmente passam por processos de transformação digital. Os estudos utilizaram uma combinação de métodos para coleta de dados: observação participativa, análise de documentos e entrevistas com funcionários.

Os resultados indicam que conceitos da indústria 4.0 alteram os processos e humanos, máquinas e recursos se comunicam de formas novas. Diante disso, às regras convencionais de saúde e segurança não tem reflexo neste contexto e precisam ser retrabalhadas. Neste ambiente os profissionais da área de segurança devem trabalhar no desenvolvimento de uma cultura se segurança pró-ativa, deixando de ser apenas treinamentos e documentações e passando a incorporar a segurança dentro da cadeia de valor, permitindo aos funcionários iniciar e liderar processos de tomada de decisão em questões de segurança.

Os riscos de digitalização industrial percebidos pelos colaboradores precisam ser identificados e abordados em formatos comunicativos adequados. Além disso, é necessário um monitoramento contínuo para novos riscos (internos e externos) para manter as diretrizes e treinamentos atualizados. Os profissionais de comunicação precisam garantir que os funcionários estejam constantemente envolvidos em processos de tomada de decisão relacionados ao risco.

\section{Artigo 3 - Human-system interaction design}

\section{requirements to improve machinery and systems safety}

Alguns sistemas de trabalho poderão evoluir para fabricação digital através dos sistemas cyber físicos, isso causa novos desafios para as áreas de engenharia de segurança e ergonomia do trabalho. Este trabalho tem como objetivo elaborar um plano para design de projetos homem-máquina que integre os conceitos de ergonomia.

Os designs ideais devem incluir todos os seres humanos, inclusive em termos demográficos, pessoas com deficiências e outras variáveis que necessitem projetos acessíveis. Sistemas de interação homem-máquina projetadas de acordo com os requisitos de design ergonômicos e de segurança e saúde ocupacional podem ajudar no desempenho do trabalho. No entanto, há desafios neste campo em termos de: Feedback, estruturas de tarefas humanas e estruturas de relacionamento.

Uma das sugestões para esse tipo de sistema são as abordagens modulares, semelhantes às indústrias de processos, no qual um sistema global é subdividido com interfaces claramente definidas. Isso permitiria avaliações de risco para módulos 


\section{REVISTA}
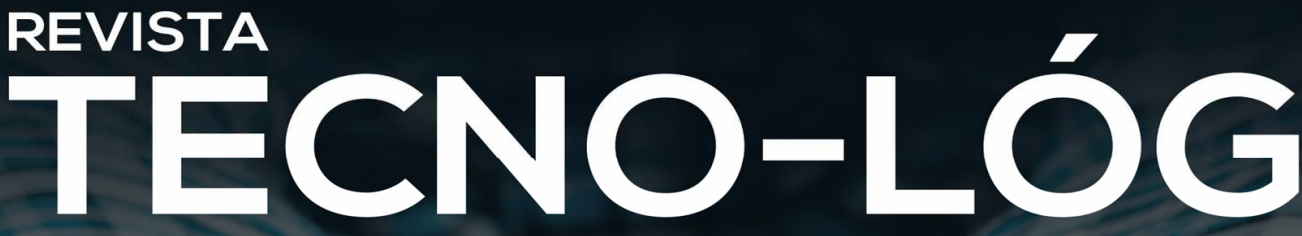

ISSN 1982-6753

Edição Especial com publicação de trabalhos selecionados do V SIGEPRO

individuais e suas combinações. Além disso, as avaliações adaptáveis e dinâmicas são promissoras, pois há a troca de informações relevantes para avaliações e estas podem ser continuamente geradas por componentes integrados no sistema. Neste caso enquanto os empregadores são responsabilizados por fornecer equipamentos de trabalho seguros aos funcionários, estes últimos são responsáveis por trabalhar de acordo com as normas.

Artigo 4 - Improvements of Machinery and Systems Safety by Human Factors Ergonomics and Safety in HumanSystem Interaction

Com a introdução das novas tecnologias da indústria 4.0 alguns sistemas de trabalho futuros permanecem inalterados, outros podem se desenvolver em sistemas cyber físicos (CPS). Este trabalho tem como objetivo informar sobre como integrar os requisitos de design da ergonomia na construção de máquinas, integração no projeto do local de trabalho e uso no nível de chão de fábrica.

O design sugerido pelos autores aborda que a construção deve contemplar: design de local de trabalho (requisitos biomecânicos, design de exibição e controle), design de software (tarefa, interação e design de informações), questões psicológicas no design de sistemas (saúde, segurança, comportamento), e Design CPS (fabricação inteligente e desafios nas avaliações de risco).

Os desafios identificados na projeção de sistemas cyber físicos são: i) desafios de feedback: Os operadores têm muitas informações e, portanto, encontram dificuldades e erros na automação difíceis e por vezes impossíveis de compensar. ii) Desafios da estrutura de tarefas humanas: As funções são retiradas dos operadores humanos pela automação, e seu trabalho é consequentemente prejudicado ou mesmo dificultado e iii)
Desafios da estrutura de relacionamento: o design centrado em tecnologia de automação leva a usos não intencionais pelos operadores.

Para melhorar a segurança e resultar em fabricação inteligente, avaliações de risco são necessárias para divulgar potenciais riscos na interação do sistema máquina em relação às tarefas humanas, hardware e software, bem como todos os processos do sistema. Uma solução para esse problema seria a implementação de sistemas CPS através de módulos. O sistema geral é separado em módulos com interfaces claramente identificadas. As avaliações de risco serão então organizadas para módulos individuais e suas combinações.

\section{Artigo 5 - Indoor Air-Quality Data-Monitoring System: Long- Term Monitoring Benefits}

O artigo aborda a utilização da internet das coisas (IoT) para auxiliar no monitoramento dos poluentes do ar em ambientes. A pesquisa desenvolveu um sistema de monitoramento de dados a partir da IoT. O sistema desenvolvido permite a coleta e gerenciamento de dados de diferentes medidas como: qualidade do ar, condições acústicas, iluminação e conforto térmico. Nove parâmetros são usados para medir condições internas.

O resumo dos resultados do nível de concentração média de poluentes é carregado e armazenado constantemente (a cada 15min), o que permite que todos os usuários possam revisar e usar os resultados estatísticos periódicos para avaliação regular da dos índices de saúde e segurança ocupacional neste ambiente.

Os resultados da pesquisa mostraram que as verificações realizadas regularmente podem distorcer resultados e não garantir a saúde e a segurança dos funcionários. Isso comprova que os critérios de inspeção, realizados em pleno respeito à regulamentação, ainda não garantem a saúde e a segurança dos 


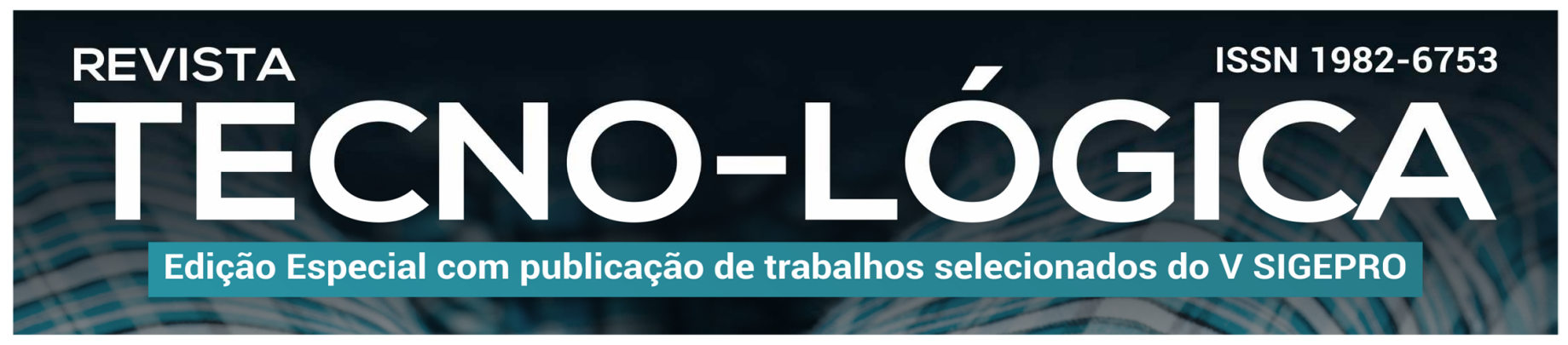

funcionários. O sistema permite o monitoramento constante para que gestores possam tomar medidas para melhorar as condições de trabalho internas.

\section{Artigo 6 - Negative influences of the 4th industrial revolution on} the workplace: towards a theoretical model of entropic citizen

\section{behavior in toxic organizations}

$\mathrm{O}$ artigo tem como incumbência mostrar como as organizações são afetadas pelas formas tóxicas de liderança, provocadas por pressões de rápida mudança industrial, automação e explosão das pressões advindas da $4^{\mathrm{a}}$ revolução industrial.

Entre os resultados os autores abordam casos de comportamento tóxico de cidadania organizacional na Volkswagen. Neste caso os engenheiros distorceram os testes de emissões de diesel por falta de tempo.

Um segundo exemplo deste mesmo gênero é a crise na Boeing. Os recentes acidentes da aeronave 737 Max, com perda completa de vidas de passageiros e tripulantes em ambos os casos, são resultados de uma liderança que tornou-se tóxica devido às pressões da concorrência industrial, provocadas por avanços implacáveis na automação aeronáutica e pela busca de lucros da empresa. Assim como na crise da VW houve a pressão para ir além do limite e comprometer etapas.

Mais um exemplo relatado pelos autores é A Body Shop, uma empresa que foi fundada no ativismo social e nos princípios das mudanças sociais e ambientais. No entanto, a liderança da empresa criou um clima tóxico e os princípios de criação foram distorcidos.

Depois de analisar estes casos como conclusão os autores afirmam que as pressões na concorrência entre empresas, provocadas pelas rápidas mudanças da $4^{\mathrm{a}}$ revolução industrial, promoveram formas tóxicas de liderança nas organizações, os trabalhadores levam os funcionários a trabalhar 24 horas por dia, 7 dias por semana e, a tentar demonstrar em formas extremas que são dignos de emprego contínuo. A gestão e as partes interessadas precisam estar atentas à liderança tóxica e evitar recrutar líderes com estes perfis. "Medidas corretivas e prescritivas precisam ser tomadas pela gestão através da implementação de programas de treinamento especificamente projetados para aliviar o estresse".

Artigo 7 - New and emerging occupational risks (NER) in industry 4.0: Literature review

Este artigo tem como objetivo revisar a literatura, para identificar os riscos laborais causados pelo uso das novas tecnologias habilitadoras da indústria 4.0 e conhecer o que já foi investigado e quais os aspectos ainda são desconhecidos.

Os bancos de dados utilizados foram Scopus, EBSCO, PubMed, Scielo, Redalyc, Science Direct, MedLine, e revistas como Ciência da Segurança e/ou coleções de trabalhos de congressos e documentos que abordassem o tema. As tecnologias consideradas são: Big data, realidade aumentada, manufatura aditiva, robôs autônomos, internet das coisas, computação em nuvem, sistema cyber securIty, sistemas integrados, simulação.

Foram identificados 12 documentos que abordavam o tema selecionado. Como resultados os autores afirmam que pouca importância vem sendo dada ao tema. Por ser um assunto emergente, a maioria dos trabalhos aborda a insuficiência de informações dadas pela Administração de Segurança e Saúde Ocupacional (OSHA) em relação às práticas a serem adotadas para identificação dos riscos ocupacionais. Não há informações suficientes para identificar as características dos novos riscos e diferenciá-los dos riscos tradicionais.

De forma geral os autores afirmam que incluir as tecnologias aos processos irá propiciar a aparição de novos riscos 


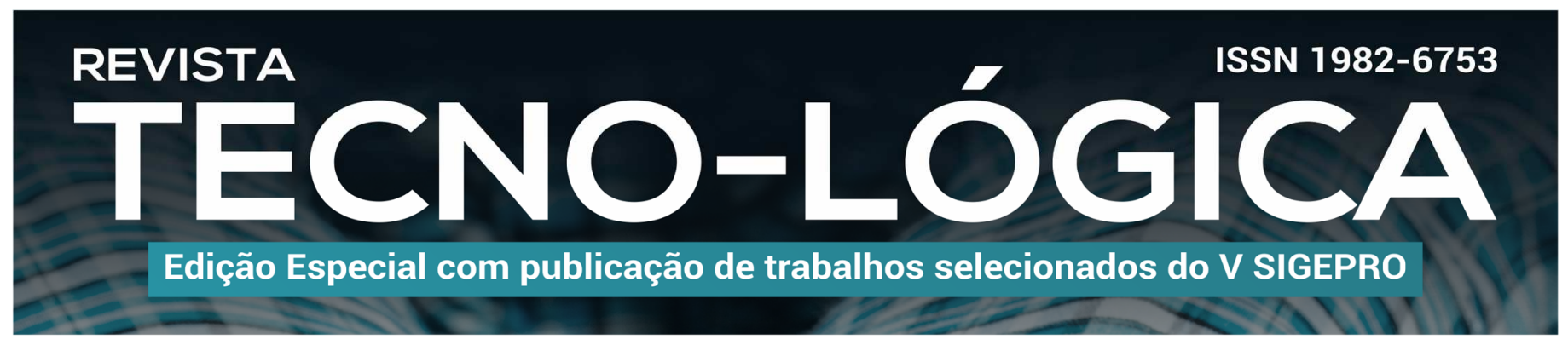

laborais, porém não há informações suficientes para identificar, quantificar e propor soluções para eles. Além disso, o uso das tecnologias também trará riscos de segurança de dados e sustentabilidade financeira do sistema.

Artigo 8 - The efficacy of industrial safety science constructs for addressing serious injuries \& fatalities (SIFs)

Este trabalho tem como objetivo agrupar as informações existentes sobre a segurança do trabalho no Reino Unido, nos últimos 30 anos, para verificar se alguma lição aprendida poderia ser aplicada no futuro. As principais questões que nortearam o trabalho são (1) qual o impacto que as abordagens defendidas tiveram nas estatísticas de lesões; (2) qual é a qualidade da ciência por trás dos efeitos positivos e (3) para onde vamos a seguir à medida que nos aprofundamos no século 21 ?

A maioria das iniciativas de segurança são monitoradas posteriormente aos incidentes e medidas em torno de número ou taxa de incidentes de acidentes e lesões. Para examinar o impacto das iniciativas anteriores e verificar o atual estado o banco de dados utilizado foi RIDDOR, que possui informações sobre o assunto desde 1974 até 2018. Os acidentes são divididos em escalas como lesões graves, fatalidades e deficiências temporárias.

Há no país uma política de construção da cultura de segurança, envolvendo o engajamento de gestores e colaboradores em iniciativas comprovadas, como: avaliação contínua da cultura de segurança, otimização dos processos de segurança comportamental, desenvolvimento de sistemas de gerenciamento de segurança eficaz e organizações que gerenciam o sistema de desempenho em Saúde e Segurança através de relatórios anuais.

Um aspecto importante encontrado nos resultados é que não houve redução significativa no número de acidentes, tanto as lesões graves e fatalidades (morte ou invalidez) quanto nas deficiências temporárias. As iniciativas de segurança introduzidas nos últimos 32 anos parecem ter feito muita pouca diferença para os números do Reino Unido. Certamente, as estratégias existentes não estão reduzindo os incidentes, algo novo é necessário ou as estratégias existentes precisam ser mudadas.

Essas observações ajudam levantam as seguintes questões: até que ponto essas novas tecnologias irão alterar os parâmetros da cultura de segurança? Como os processos de segurança comportamental serão adaptados para lidar com a influência das tecnologias digitais? Os sistemas existentes não são bons. Logo no novo cenário eles precisam ser adaptados ou renovados? que tipo de controles de risco serão necessários? Como serão esses métodos de controle de risco? Será que a cultura de segurança construirá fragmento em ferramentas e metodologias específicas da indústria 4.0 ou será validado modelos universais? Claramente, em muitos domínios da segurança haverá muitos desafios para os paradigmas existentes da $4^{\mathrm{a}}$ revolução industrial e todas as perguntas ainda estão sem respostas.

\section{Discussões}

É possível identificar que os artigos human-system interaction design requirements to improve machinery and systems safety, improvements of machinery and systems safety by human factors ergonomics and safety in human-system interaction, indoor air-quality data-monitoring system: long-term monitoring benefits, negative influences of the 4th industrial revolution on the workplace: towards a theoretical model of entropic citizen behavior in toxic organizations não especificam população para quais seus modelos são propostos. Não há discussão se podem ser aplicados a diferentes tipos e tamanhos de organização ou se aplicam a apenas um. 


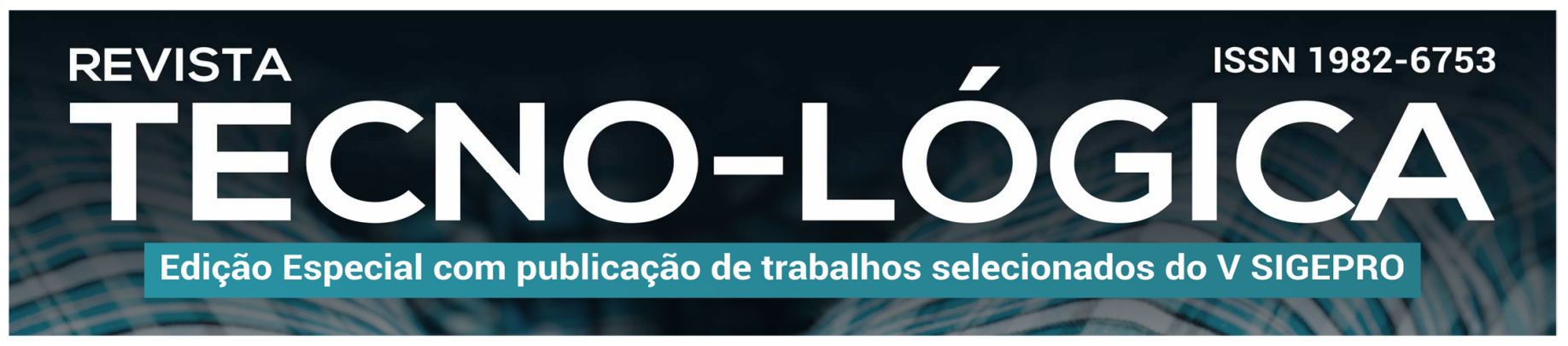

The efficacy of industrial safety science constructs for addressing serious injuries \& fatalities (SIFs) é um artigo no qual os autores iniciam falando que vão criar uma visão sobre o impacto da indústria 4.0 na área de segurança, à medida que vai evoluindo eles apenas vão contextualizando à área de segurança e finalizam sem grandes conclusões da relação existente entre segurança e indústria 4.0, deixando apenas perguntas em aberto.

$\mathrm{O}$ artigo New and emerging occupational risks (NER) in industry 4.0: Literature review não faz uma definição clara do que é risco no contexto abordado pelo artigo. Os autores não definem e não exemplificam o que é risco, apenas afirmam que não há informações suficientes para identificar os riscos e que há pouca discussão em torno do assunto.

Os resultados encontrados pelos autores estão divididos entre vantagens da implementação das tecnologias e desvantagens. Para alguns autores a indústria 4.0 pode ser explorada positivamente pela criação de robôs para tarefas perigosas, obtenção de dados mais preciso, monitoramento constante, maior quantidade de dados, resultados mais precisos, alocação de tarefa mais facilmente entre outras [6 - 13]. Por outro lado, esse ambiente também traz desvantagens como maior cobrança, dificuldade de identificação de riscos e proposição de melhorias, substituição de mão de obra, maior índice de stress entre os funcionários, entre outros [8 - 9].

\section{Conclusões}

Os resultados confirmam o que já havia sido mencionado pelos autores Issamar e Roberto [3], de que o tema indústria 4.0 vem crescendo no número de publicações no que diz respeito a conceitualização, aplicações em empresas de diferentes setores e tamanhos, oportunidades e desafios na sua adoção, mas ainda há uma baixa importância sendo dada na área de gestão da saúde e segurança no trabalho. O pouco número de artigos diretamente relacionados ao tema corrobora a afirmação dos autores.

Por ser um tema considerado ainda emergente há necessidade de mais pesquisas para responder como as tecnologias da indústria 4.0 afetam a área de segurança do trabalho. O que fica evidenciado pelos resultados obtidos é que não se tem ainda uma abordagem definida de qual o impacto das tecnologias da indústria 4.0 para a área de segurança do trabalho. Nos estudos selecionados alguns autores apontam vantagens, outros falam sobre desvantagens e há os que ainda não discutem esse tópico. Além disso, os estudos revisados apresentam muitas perguntas ainda sem respostas e que podem ser exploradas.

Diante disso, o presente artigo traz contribuições apontando que o cenário ainda é muito vago, há poucas pesquisas que abordam o tema tecnologias da indústria 4.0 na área de segurança do trabalho e há mais perguntas do que respostas. Além disso, há carência de estudos que relatam aplicações práticas das tecnologias da indústria 4.0 e como isso impacta na área de saúde e segurança do trabalho, o que oferece uma lacuna para desenvolvimento de novos estudos focados neste tema, que apresenta relevância devido ao aumento do interesse no uso destas tecnologias.

\section{IMPACT OF DIGITAL TECHNOLOGIES OF} INDUSTRY 4.0 ON OCCUPATIONAL HEALTH AND

\section{SAFETY: AN ANALYSIS THROUGH SYSTEMATIC LITERATURE REVIEW}

ABSTRACT: The new industry 4.0 paradigm is revolutionizing organizations and has an impact on different sectors. Looking at 


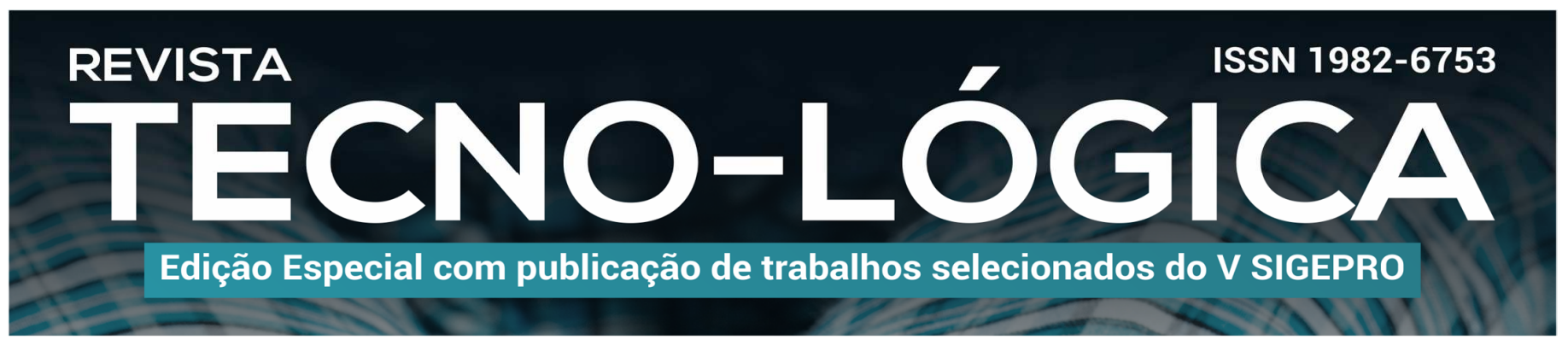

this spectrum, this work aims to verify the impact of the intelligence, 78 , 218-235. Disponível em: technologies of industry 4.0 in the area of health and safety at work. A systematic literature review was carried out, seeking to analyze the advantages, disadvantages, and challenges of using the technologies of industry 4.0 in the context of health and safety at work. The databases used were Web, Science, and Scopus. The documents were selected according to pre-defined criteria. The results indicate that the scenario is still very vague. Few studies address the theme of industry 4.0 technologies in the area of work safety, highlighting a gap in research and scientific exploration.

Keywords: Occupational Health and Safety. Industry 4.0. Literature Review. https://www.sciencedirect.com/science/article/abs/pii/S0952197618302458. Acesso em 4 abr 2020.

[6] LAZZERINI, Beatrice; PISTOLESI, Francesco. Artificial Bee Colony Optimization to Reallocate Personnel to Tasks Improving Workplace Safety. In: International Workshop on Machine Learning, Optimization, and Big Data. Springer, Cham, 2017. p. 210-221. Disponível em: https://link.springer. com/chapter/10.1007/978-3-319-72926-8_18. Acesso em 18 mar 2020.

[7] WICHTL, Michael et al. Improvements of Machinery and Systems Safety by Human Factors, Ergonomics and Safety in Human-System Interaction. In: Congress of the International Ergonomics Association. Springer, Cham, 2018. p. 257-267. Disponível em: https://link.springer.com/chapter/10.1007/978-3-31996089-0_28. Acesso em 03 mar 2020.

[8] COLDWELL, David et al. Negative Influences of the 4th Industrial Revolution on the Workplace: Towards a Theoretical Model of Entropic Citizen Behavior in Toxic Organizations. International journal of environmental research and public health, v. 16, n. 15, p. 2670, 2019. Disponível em: https://www.mdpi.com/16604601/16/15/2670. Acesso em 12 fev 2020.

[1] IBGE- Instituto Brasileiro de Geografia e Estatística. Coordenação de População e Indicadores Sociais. Estatísticas da saúde: assistência médicosanitária, 2009. IBGE, 2010.

[2] MATTOS, Ubirajara; MÁSCULO, Francisco. Higiene segurança do trabalho. Elsevier Brasil, 2011.

[3] ISSAMAR, Favela Herrera Marie Karen; ROBERTO, Romero López. New and Emerging Occupational Risks (NER) in Industry 4.0: Literature Review. In: 2019 7th International Engineering, Sciences and Technology Conference (IESTEC). IEEE, 2019. p. 394-399. Disponível em: https://ieeexplore.ieee.org/ abstract/document/8943671/. Acesso em 20 mar 2020.

[4] ONU News. Acidentes de trabalho matam 2,3 milhões de pessoas por ano no mundo, diz OIT, Agência Brasil, 2018. Disponivel em: http://agenciabrasil.ebc.com.br/internacional/noticia/2017-04/acidentes-detrabalho-matam-23-milhoes-de-pessoas-por-ano-no-mundo-diz. Acesso em $23 \mathrm{fev}$ 2020.

[5] MUHURI, P. K.,SHUKLA, A. K., ABRAHAM, A. (2019). Industry 4.0: A bibliometric analysis and detailed overview. Engineering applications of artificial

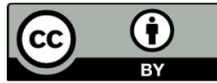




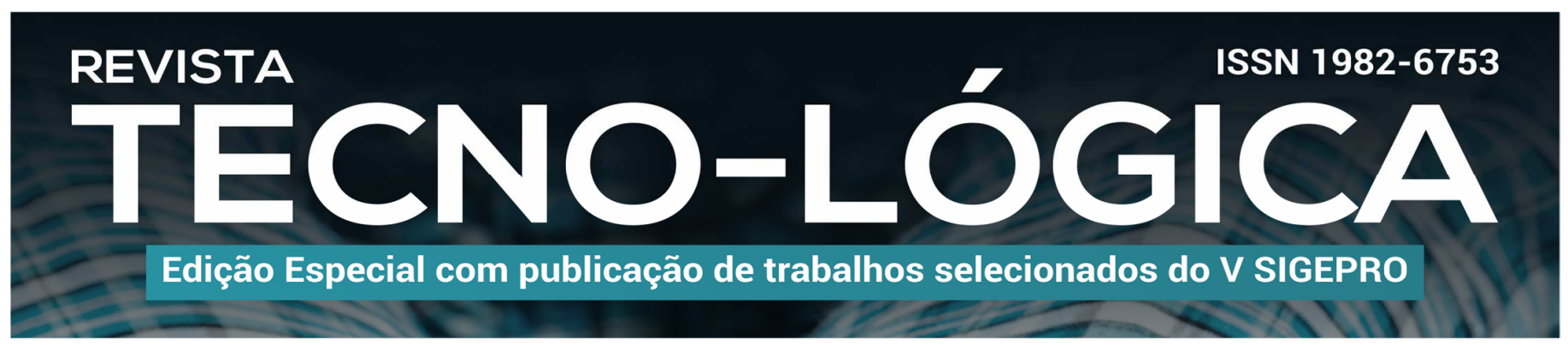

International Professional Communication Conference (ProComm). IEEE, 2019. p. 218-225. Disponível em: https://ieeexplore.ieee.org/abstract/document/ 8804592. Acesso em 23 mar 2020.

[13] COOPER, M. Dominic. The efficacy of industrial safety science constructs for addressing serious injuries \& fatalities (SIFs). Safety Science, v. 120, p. 164 178, 2019. Disponível em: https://www.sciencedirect.com/science/article/pii/ S0925753518320071. Acesso em 18 fev 2020.

[14] SUN, Shengjing et al. Indoor Air-Quality Data-Monitoring System: LongTerm Monitoring Benefits. Sensors, v. 19, n. 19, p. 4157, 2019. Disponível em: https://www.mdpi.com/1424-8220/19/19/4157. Acesso em 30 jan 2020. 